\title{
REVISTA
}

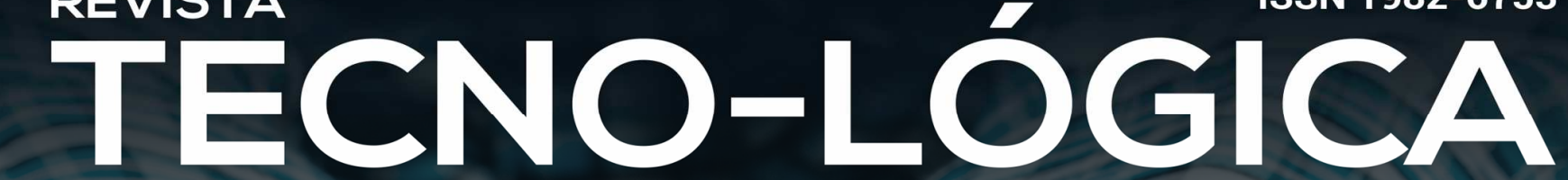

Edição Especial com publicação de trabalhos selecionados do V SIGEPRO

\section{INDICADORES NO SETOR DE SUPRIMENTOS: UM ESTUDO EXPLORATÓRIO NAS INDÚSTRIAS FABRICANTES DE EMBALAGENS FLEXÍVEIS}

\author{
Eduardo Bakkar Reckers ${ }^{\text {* }}$, Lucas Schmidt Goecks ${ }^{2}$, Ismael Becker Gomes ${ }^{2}$, Elpidio Oscar Benitez Nara ${ }^{3}$

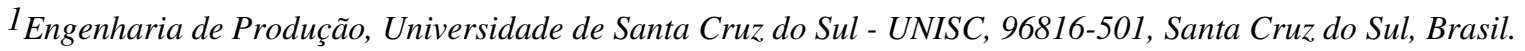 \\ ${ }^{2}$ Engenharia de Produção e Sistemas, Universidade do Vale do Rio dos Sinos - UNISINOS, 93022-750, São Leopoldo, Brasil. \\ ${ }^{3}$ Sistemas e Processos Industriais, Universidade de Santa Cruz do Sul - UNISC, 96816-501, Santa Cruz do Sul, Brasil.
}

*E-mail:dudubakkar@hotmail.com

Recebido em: 17/09/2020

Aceito em: 06/11/2020

DOI: $10.17058 /$ tecnolog.v2i0.15616

\section{RESUMO}

Diversas empresas encontram dificuldades para maximizar o potencial de sua cadeia de suprimentos, pois falham em desenvolver um método de avaliação eficaz. Diante dessa problemática e da necessidade de se mensurar efetivamente a cadeia de suprimentos, este estudo tem como objetivo identificar os indicadores de desempenho. A pesquisa estruturou-se a partir de uma revisão bibliográfica e de um questionário para identificação dos principais indicadores da cadeia de suprimentos, que foi enviado para empresas de embalagens flexíveis do RS. A partir dos resultados e cálculos dos KPI, FCS e PVF, foram definidos rankings conforme o questionário. Para os KPI, os indicadores de maior importância foram: custo do produto vendido, OTD e qualidade do produto adquirido. Tratando-se dos FCS, foram definidos como mais importantes os custos operacionais, acuracidade do atendimento do pedido e o cumprimento de especificações técnicas de qualidade. Referente aos PVF, as melhores colocações foram qualidade, flexibilidade e custos. Por fim, este estudo contribui com a proposição de indicadores-chave de desempenho, a partir da literatura e apoiado por gestores de cadeias de suprimentos.

Palavras-chave: Suprimentos. Indicadores. Embalagens Flexíveis. Survey.

\section{Introdução}

A habilidade de entregar o produto certo, na hora certa e com custo logístico baixo é um dos objetivos e medidas de desempenho da cadeia de suprimentos [1]. Portanto, torna-se imprescindível que o desempenho da cadeia de suprimentos seja controlado. Para tal, o desenvolvimento de um sistema de medição de desempenho requer uma seleção apropriada dos indicadores. 


\section{REVISTA}

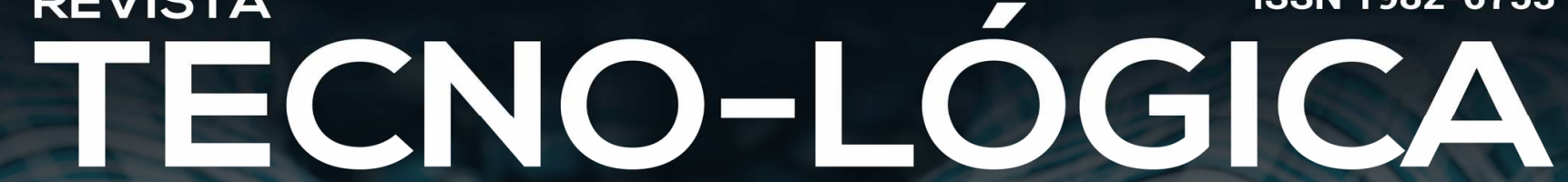

Edição Especial com publicação de trabalhos selecionados do V SIGEPRO

Entretanto, com a existência de diversos indicadores, torna-se complexa a interpretação de cada um deles, gerando incertezas por parte da organização quanto a real estratégia proposta [2].

A gestão da cadeia de suprimentos pode depender da situação da instituição e também da estrutura adotada pela mesma. Há diversas percepções quanto à efetividade da gestão e a localização da empresa que influenciam no modo com que ela entende e maneja seus negócios. Essas evidências demonstram que as organizações necessitam adquirir mais conhecimento para perceber que uma maior efetividade na gestão da cadeia de suprimentos pode gerar vantagens competitivas e impulsionar seu desempenho [3].

Muitas empresas não conseguem maximizar o potencial de sua cadeia de suprimentos porque falham em desenvolver um método de avaliação de desempenho. Os principais problemas encontrados na avaliação de desempenho são [4]: inconsistências nas medidas e métricas de desempenho, falha ao representar um conjunto de medidas financeiras e não-financeiras de forma equilibrada, um grande número de métricas, não observando quais são as mais importantes (KPI - Key Performance Indicators), falha em alinhar a estratégia da organização com a medição adotada, tendência predeterminada nas medições e a falta de uma visão global do sistema. Diante dessa problemática e da necessidade de mensurar efetivamente a cadeia de suprimentos, destaca-se a questão de pesquisa: quais são os indicadores de desempenho mais utilizados na gestão do setor de suprimentos das indústrias de embalagens flexíveis do Rio Grande do Sul?

Para tal questionamento, o objetivo deste estudo consiste em identificar os indicadores de desempenho da cadeia de suprimentos nas indústrias de embalagens flexíveis do Rio Grande do Sul. Portanto, os objetivos específicos foram: determinar os Pontos de Vista Fundamentais (PVF), os Fatores Críticos de Sucesso (FCS) e os Indicadores-Chave de Desempenho (KPI). Uma survey com indústrias fabricantes de embalagens flexíveis do Rio Grande do Sul forneceu dados necessários para a análise e modelagem dos PVF, FCS e KPI.

O desenvolvimento da pesquisa ocorreu em cinco etapas, sendo que cada etapa representa uma das seções deste artigo. $\mathrm{Na}$ introdução foram apresentados a problemática, a justificativa, as questões de pesquisa, os objetivos e a relevância prática. No referencial teórico pesquisou-se a conceituação de termos para o desenvolvimento deste estudo. $\mathrm{Na}$ estruturação do método de pesquisa foram demonstradas as diretrizes empregadas para responder os objetivos propostos. A partir desse método, a identificação dos indicadores e posterior avaliação por meio da survey foram desenvolvidos. Por fim, os resultados foram analisados e discutidos, juntamente com as conclusões geradas a partir da pesquisa.

\section{Referencial teórico}

\subsection{Cadeia de Suprimentos}

Segundo Mentzer et al. [5], uma cadeia de suprimentos é definida como o conjunto de três ou mais entidades diretamente envolvidas nos fluxos dos produtos, serviços, finanças e/ou informações fornecidas para um cliente. O foco está na cooperação, confiança e reconhecimento. Quando gerida da maneira correta, proporciona ao processo um desempenho melhor, como um todo, do que quando comparada etapa por etapa [6].

Para melhor desempenho da cadeia de suprimentos é necessário que a empresa diminua custos e aumente a eficiência 


\section{REVISTA}

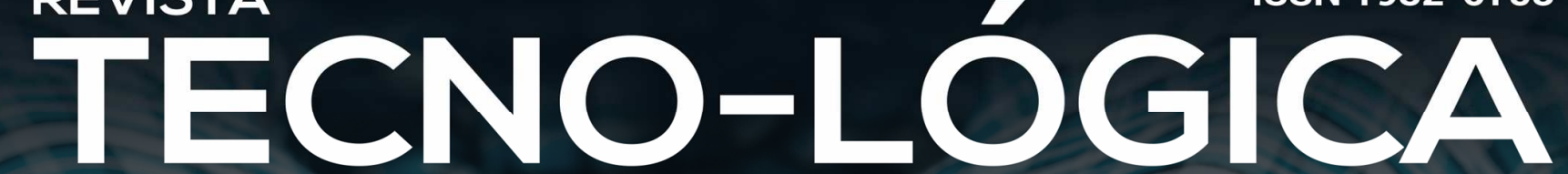

\section{Edição Especial com publicação de trabalhos selecionados do V SIGEPRO}

de atendimento a seus clientes. Nesse sentido, torna-se relevante mensurar pontos essenciais da cadeia para que intervenções assertivas sejam feitas. A avaliação do desempenho é imprescindível no controle de processos. Se torna importante em definir objetivos, monitorar e avaliar a performance para guiar futuras ações. A definição mais comum de medição de desempenho é a do processo de quantificação da eficiência e efetividade da ação [7].

\subsection{Indicadores de desempenho}

$\mathrm{O}$ uso de indicadores de desempenho tem o intuito de melhorar o entendimento do processo, identificar gargalos, desperdícios, problemas e oportunidades de melhorias, tomar decisões baseadas em fatos, criar condições para o desenvolvimento da empresa, monitorar o atendimento das expectativas do cliente e facilitar a comunicação [8]. Uma vez que as medições de desempenho da cadeia de suprimentos são feitas adequadamente, os gestores precisam identificar os KPI a serem melhorados. No entanto, é difícil identificar a relação intrínseca entre diferentes KPI e a prioridade que cada um deve receber. A definição da prioridade dada a cada um desses parâmetros é um gargalo para muitas empresas devido aos seus esforços em aprimorar a gestão da cadeia de suprimentos [9]. Os gestores normalmente definem os KPI de acordo com suas necessidades e experiências práticas. Considerando o quão complexa é a cadeia de suprimentos, a metodologia pode variar entre diversas ferramentas [9].

Com a identificação dos KPI essenciais ao processo tornase possível apontar os FCS. A técnica dos FCS é uma aproximação abstrata que identifica fatores que tenham alto impacto no sucesso, porém, esse não é garantido [10]. Os FCS são definidos como limitados números de fatores que, caso sejam utilizados e seus resultados sejam satisfatórios, irão garantir uma vantagem competitiva de sucesso para as organizações [11]. Portanto, para uma plena gestão da cadeia de suprimentos, o entendimento das relações dos FCS pode conduzir aos PVF. Esses explicitam os valores que o tomador de decisões considera como importantes em determinado contexto, definindo ao mesmo tempo as características de todas as ações que serão de interesse [12]. A estruturação dos desdobramentos para uma gestão plena da cadeia de suprimentos é apresentada na Figura 1.

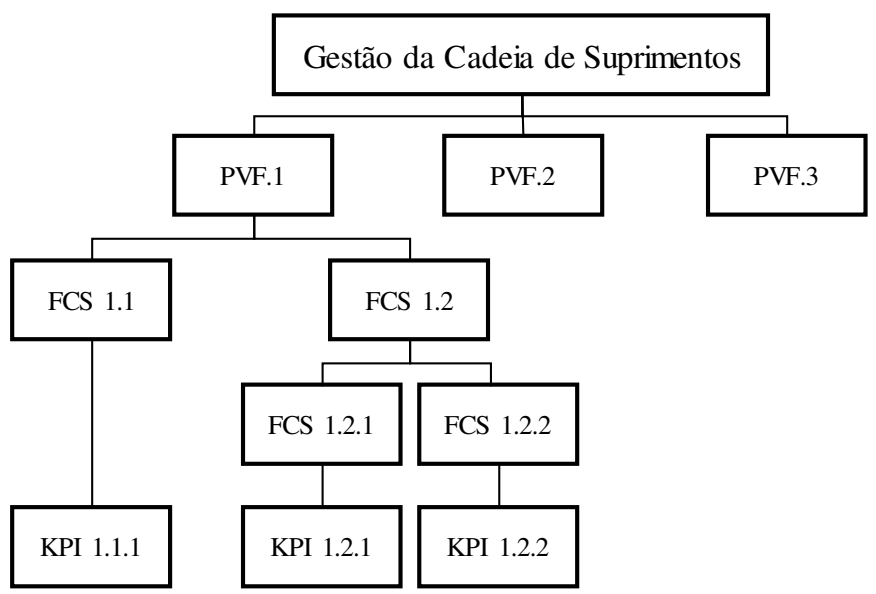

Figura 1- Esquematização de modelagem para mensuração dos indicadores [13].

\subsection{Estruturação da Modelagem e Taxas de Substituição}

A estruturação da modelagem envolve a utilização de ferramentas que permitam avaliar quantitativamente cada critério. Dessa forma, é preciso realizar a definição de taxas de substituição entre os elementos do modelo, a fim de identificar as diferenças de importância entre cada um [14]. É essencial que nessa fase seja feita a construção de funções de valor capazes de promover a medição dos atributos desejáveis de cada alternativa, os quais são estabelecidos conforme os critérios considerados como relevantes 


\section{REVISTA}

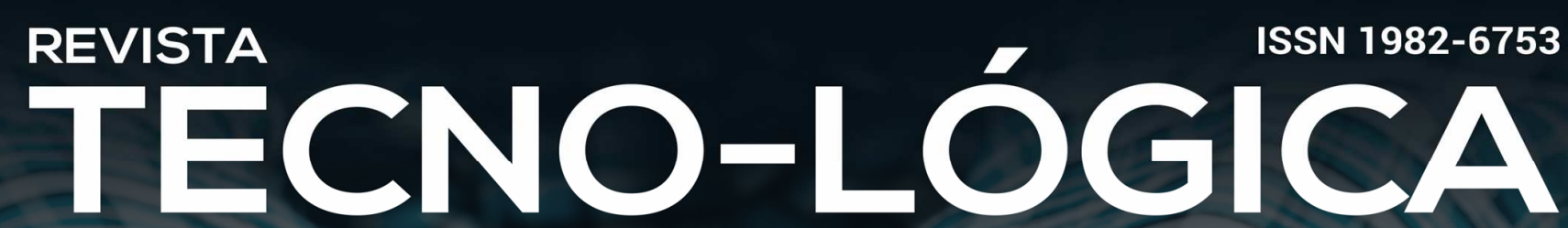

\section{Edição Especial com publicação de trabalhos selecionados do V SIGEPRO}

para a decisão [15]. Assim que as funções de valor e as taxas de substituição estiverem estruturadas, faz-se necessária a construção de uma equação global, capaz de checar efetivamente a performance de cada alternativa [14].

Depois de estabelecidas as taxas de substituição, o cálculo das taxas globais dos KPI é feito através do produto das taxas locais de substituição dos KPI, localizados nos níveis superiores da árvore de decisão [13]. Esse processo foi realizado com todos os elementos que fazem parte da árvore de decisão, sendo que a modelagem que mensura o desempenho dos indicadores do setor de suprimentos será fruto dos resultados da composição final das taxas de substituição.

\section{Método de trabalho}

Este trabalho se desenvolveu a partir de uma pesquisa exploratória, visando ter uma maior profundidade no tema em questão. Quanto aos procedimentos técnicos, classifica-se como pesquisa bibliográfica, devido a necessidade de se buscar publicações científicas e coletar dados dos entrevistados [16]. A esquematização da condução da pesquisa está representada na Figura 2.

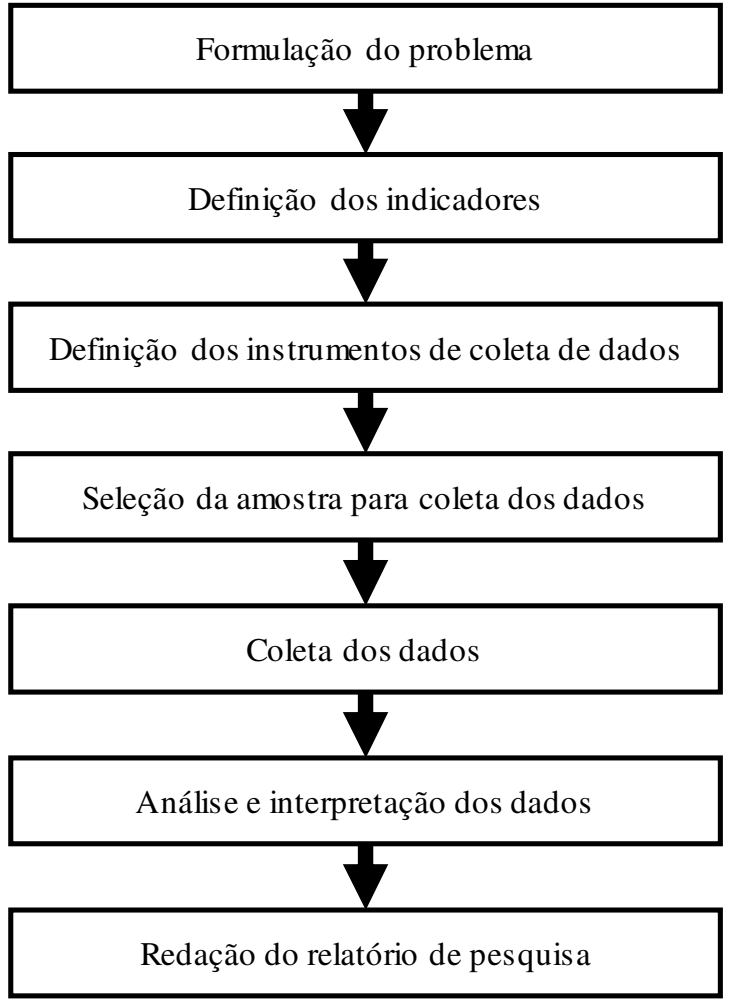

Figura 2 - Esquematização da pesquisa [16].

O desenvolvimento desta pesquisa foi dividido em duas partes. A primeira consiste na formulação do problema, na definição de indicadores, na determinação de métodos para a coleta dos dados e na seleção da amostra a ser analisada. Enquanto que, na segunda etapa, são efetuados a coleta e análise desses dados e a redação do relatório acerca dos achados da pesquisa.

Na primeira parte da pesquisa, após a definição do problema de pesquisa, a literatura foi mapeada acerca dos indicadores de desempenho do setor de suprimentos. Foram pesquisados na literatura os KPI mais utilizados no setor de suprimentos e posterior estruturação e agrupamento em FCS e PVF. Com a estrutura construída, tornou-se possível a definição 


\section{REVISTA}

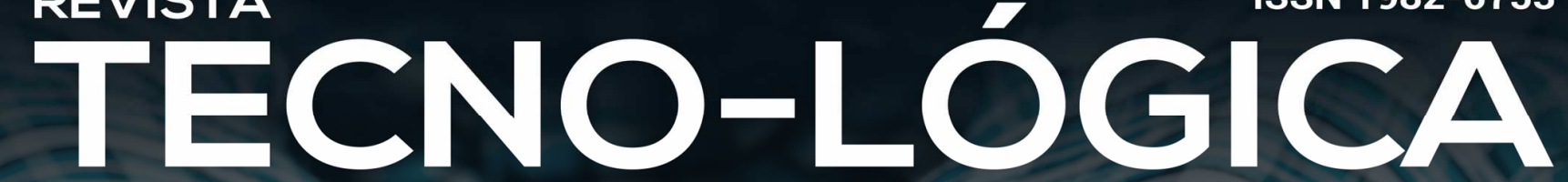

\section{Edição Especial com publicação de trabalhos selecionados do V SIGEPRO}

dos instrumentos para coleta dos dados referente aos indicadores que as empresas utilizam em seus setores de suprimentos. Essa coleta de dados foi desenvolvida com a aplicação de uma survey (questionário) direcionada a empresas do setor de embalagens flexíveis do Estado do Rio Grande do Sul.

Após a aplicação do questionário, os resultados foram coletados, tabulados e analisados. De acordo com Carnevalli e Miguel [17], os questionários classificados como estruturados não disfarçados são aqueles em que a pessoa reconhece o intuito da pesquisa, a partir de questionamentos diretos e objetivos. Esse foi empregado com o intuito de identificar quantitativamente a influência dos KPI na empresa do respondente. Em complemento, Thayer-Hart et al. [18] destacam a importância de se padronizar as perguntas; para este estudo foram selecionadas perguntas fechadas, pois são estruturadas a partir de um checklist de opções, as quais o entrevistado responde de acordo com uma escala. $\mathrm{O}$ software Sphinx foi utilizado como ferramenta de operacionalização da survey para questionar os tomadores de decisão (gerentes das cadeias de suprimentos) quanto ao nível de importância de cada indicador, a partir de uma escala padronizada. $\mathrm{Na}$ sequência, as taxas de substituição e taxa global foram calculadas para a verificação da acuracidade do ranking dos KPI, FCS e PVF. Ao final, constatações foram elaboradas acerca dos resultados.

\section{Resultados}

\subsection{Definição dos KPI, FCS e PVF}

Partindo da necessidade de mensuração do desempenho com indicadores na cadeia de suprimentos, o Supply Chain Council [19] - em português, Conselho de Cadeia de Suprimentos
- definiu cinco objetivos de desempenho, sendo eles: qualidade, rapidez, confiabilidade, flexibilidade e custo. Esses cinco pilares são suportados por Ambe [20], que os têm como padrão para se avaliar uma cadeia de suprimentos. A seguir, nas Tabelas 1 e 2, encontram-se as relações entre atributos e fatores críticos de desempenho.

Tabela 1 - KPI a serem avaliados numa cadeia de suprimentos [20].

\begin{tabular}{|c|c|}
\hline Atributo & Key Performance Indicators \\
\hline \multirow{6}{*}{ Qualidade } & Cumprimento das normas de desempenho de qualidade; \\
\hline & Defeito detectado de unidade produzida por unidade \\
\hline & comprada; \\
\hline & Prêmios de qualidade; \\
\hline & Produtos por unidade vendida; \\
\hline & Aptidão para o uso. \\
\hline \multirow{2}{*}{ Flexibilidade } & Tempo de resposta da cadeia de suprimentos; \\
\hline & Flexibilidade de produção. \\
\hline \multirow{2}{*}{ Custo } & Medidas de custo dentro da organização; \\
\hline & Custo total do gerenciamento da cadeia de suprimentos. \\
\hline \multirow{4}{*}{$\begin{array}{l}\text { Confiabilidade } \\
\text { no Fornecedor }\end{array}$} & Eficácia dos fornecedores; \\
\hline & Identificação dos fornecedores; \\
\hline & Melhoria na comunicação com fornecedores; \\
\hline & Melhoria no gerenciamento de riscos com fornecedores. \\
\hline \multicolumn{2}{|l|}{ Capacidade de } \\
\hline resposta & Tempo de entrega do pedido. \\
\hline
\end{tabular}

Prazo de entrega $\quad$ Cumprimento das encomendas no prazo;

Entrega livre de danos;

da encomenda Entrega completa conforme necessário;

Entrega atende aos requisitos dos clientes.

\begin{tabular}{ll}
\hline $\begin{array}{l}\text { Confiabilidade } \\
\text { de entrega do }\end{array}$ & Desempenho de entrega; \\
produto & Entregas pontuais; \\
& Cumprimento perfeito dos pedidos. \\
\hline \multirow{2}{*}{ Gestão de ativos } & Tempo de ciclo de caixa; \\
& Quantidade de dias no estoque; \\
& Giro dos ativos.
\end{tabular}

Giro dos ativos. 


\section{REVISTA}

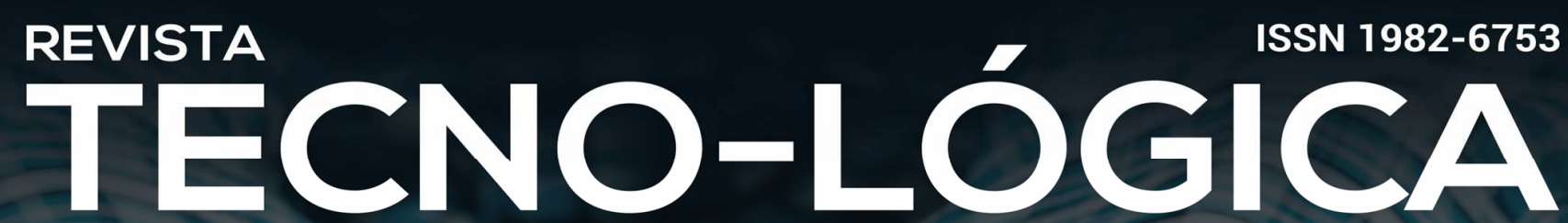

\section{Edição Especial com publicação de trabalhos selecionados do V SIGEPRO}

Tabela 2 - Atributos de desempenho em relação aos PVF [19].

\begin{tabular}{|c|c|c|c|c|c|}
\hline Atributos de desempenho & $\# 1$ & $\# 2$ & \#3 & \#4 & $\# 5$ \\
\hline Desempenho da entrega & $\mathrm{X}$ & & & & \\
\hline Taxa de ocupação & $\mathrm{X}$ & & & & \\
\hline Pedido Perfeito & $\mathrm{X}$ & & & & \\
\hline Lead time do pedido & & $\mathrm{X}$ & & & \\
\hline Tempo de resposta da cadeia de suprimentos & & & $\mathrm{X}$ & & \\
\hline Flexibilidade da produção & & & $\mathrm{X}$ & & \\
\hline Custo total de SCM & & & & $\mathrm{X}$ & \\
\hline Custo dos produtos vendidos & & & & $\mathrm{X}$ & \\
\hline Produtividade com valor adicionado & & & & $\mathrm{X}$ & \\
\hline Custo da garantia ou de processamento de retornos & & & & $\mathrm{X}$ & \\
\hline Tempo de ciclo de caixa & & & & & $\mathrm{X}$ \\
\hline Estoque em dias de suprimento & & & & & $\mathrm{X}$ \\
\hline Giro do ativo & & & & & $\mathrm{X}$ \\
\hline
\end{tabular}

\#1 Confiabilidade; \#2 Responsabilidade; \#3 Flexibilidade; \#4 Custos; \#5 Ativos.

Ambe [20] destaca que os KPI, apresentados na Tabela 1, podem ser desdobrados, tendo em vista que são o modo de mensuração dos FCS. Sendo assim, na Tabela 3 pode ser observada a árvore de decisão estruturada a partir dos dados obtidos.

Foram estipulados $16 \mathrm{KPI}$, agrupados em 12 FCS que, por fim, compõem os 5 PVF iniciais. Após a definição dos três grupos de modelagem, elaborou-se a ferramenta de pesquisa, que visa abranger todos os KPI na forma de um questionário. Esse foi enviado aos gestores das cadeias de suprimentos das empresas contatadas, visando mensurar qual a importância de cada um desses indicadores para cada organização. A fim de padronizar a pesquisa, foi definido uma escala de 0,25 ("sem importância”), 0,50 ("pouco importante"), 0,75 ("importante) até 1,00 ("muito importante"). O instrumento de pesquisa foi construído no software Sphinx (Figura 3) e as perguntas foram separadas em blocos, conforme os PVF.

\begin{tabular}{|c|c|c|c|c|c|}
\hline & $P V F$ & & FCS & & KPI \\
\hline \multirow{3}{*}{ PVF.1 } & \multirow{3}{*}{ Qualidade } & FCS.1 & $\begin{array}{l}\text { Cumprir as } \\
\text { especificações }\end{array}$ & KPI.1 & $\begin{array}{l}\text { Qualidade do } \\
\text { produto adquirido }\end{array}$ \\
\hline & & & técnicas de qualidade & & \\
\hline & & FCS.2 & $\begin{array}{l}\text { Menor número de } \\
\text { defeitos }\end{array}$ & KPI.2 & $\begin{array}{l}\text { Defeito por } \\
\text { unidade }\end{array}$ \\
\hline \multirow{4}{*}{ PVF.2 } & \multirow{4}{*}{ Flexibilidade } & FCS.3 & Sazonalidade de preço & KPI.3 & Evolução de preço \\
\hline & & FCS.4 & Prazo de pagamento & KPI.4 & $\begin{array}{l}\text { Prazo médio de } \\
\text { pagamento }\end{array}$ \\
\hline & & FCS.5 & $\begin{array}{l}\text { Valor economizado na } \\
\text { compra }\end{array}$ & KPI.5 & Saving \\
\hline & & FCS.6 & $\begin{array}{l}\text { Possibilidade de } \\
\text { alteração do pedido de } \\
\text { compra }\end{array}$ & KPI.7 & $\begin{array}{l}\text { Flexibilidade de } \\
\text { volume } \\
\text { Flexibilidade de } \\
\text { entrega }\end{array}$ \\
\hline \multirow{5}{*}{ PVF.3 } & \multirow{5}{*}{ Rapidez } & FCS.7 & $\begin{array}{l}\text { Reabastecimento do } \\
\text { estoque }\end{array}$ & KPI.8 & Ponto de pedido \\
\hline & & FCS.8 & $\begin{array}{l}\text { Tempo entre a } \\
\text { solicitação de compra }\end{array}$ & KPI.9 & Lead time \\
\hline & & & e o recebimento & & \\
\hline & & FCS.9 & Pontualidade & KPI.10 & OTD (On-Time \\
\hline & & & & & Delivery) \\
\hline \multirow[b]{2}{*}{ PVF.4 } & \multirow[b]{2}{*}{ Confiabilidade } & FCS.10 & Entrega sem avaria & KPI.11 & Devoluções \\
\hline & & FCS.11 & $\begin{array}{l}\text { Acuracidade do } \\
\text { atendimento do } \\
\text { pedido }\end{array}$ & $\begin{array}{l}\text { KPI.12 } \\
\text { KPI.13 }\end{array}$ & $\begin{array}{l}\text { Pedido perfeito } \\
\text { OTIF (On-Time in } \\
\text { Full) }\end{array}$ \\
\hline \multirow{3}{*}{ PVF.5 } & \multirow{3}{*}{ Custos } & \multirow{3}{*}{ FCS.12 } & \multirow{3}{*}{ Custos Operacionais } & KPI.14 & $\begin{array}{l}\text { Custo de } \\
\text { processamento dos } \\
\text { pedidos }\end{array}$ \\
\hline & & & & KPI.15 & $\begin{array}{l}\text { Custo de } \\
\text { manutenção do } \\
\text { estoque }\end{array}$ \\
\hline & & & & KPI.16 & $\begin{array}{l}\text { Custo do produto } \\
\text { vendido }\end{array}$ \\
\hline
\end{tabular}




\section{REVISTA}

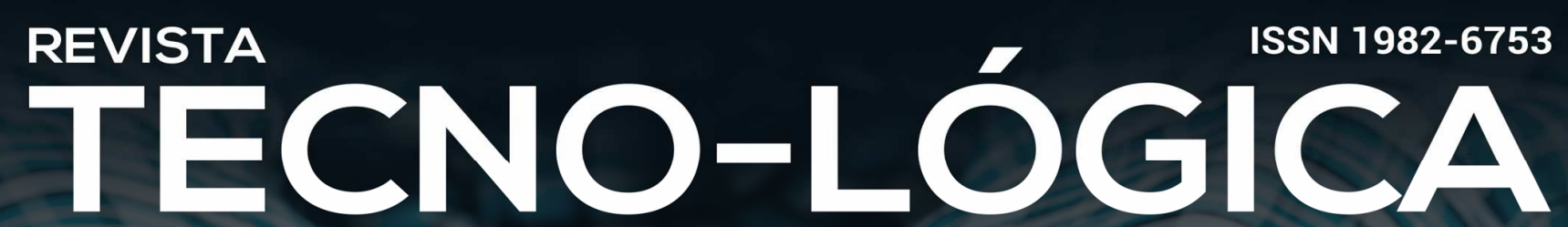

\section{Edição Especial com publicação de trabalhos selecionados do V SIGEPRO}

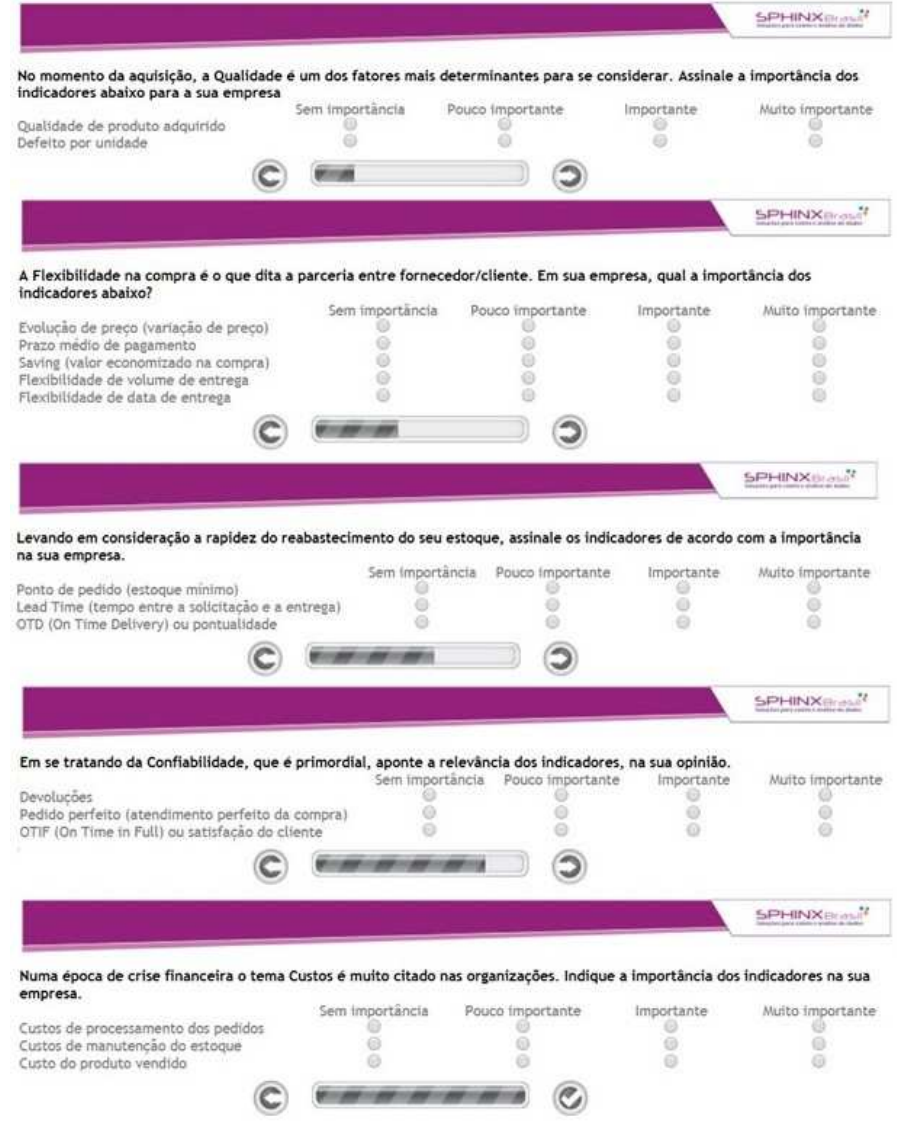

Figura 3 - Bloco de perguntas.

Quanto ao contato com as empresas, inicialmente, via telefone, certificou-se quem ocupava o cargo de gerência de suprimentos a fim de encaminhar diretamente o questionário para o respondente e explicar o objetivo da pesquisa e a forma de preenchimento da mesma. O envio do link do questionário foi efetuado através de e-mail, onde constavam o prazo limite para resposta, o tempo aproximado de preenchimento e a menção do anonimato.

\subsection{Coleta de dados e confiabilidade amostral}

Foram enviados e-mails para 46 gestores das empresas selecionadas, obtendo-se um retorno de 21 destes. Com a finalidade de sigilo das empresas, informações de caracterização foram desconsideradas. Para tratamento estatístico e validação científica, utilizou-se uma ferramenta online para cálculo amostral, aplicando os parâmetros de população e amostra respondente, onde se obteve um erro amostral de $12 \%$ a um nível de confiabilidade de $90 \%$.

\subsection{Cálculo das taxas de substituição}

Todos os dados recebidos da survey foram tabulados e agrupados na Tabela 4. Nela são indicados o número de vezes que cada KPI foi escolhido para determinado nível de importância, baseado na experiência do gestor de compras que preencheu o questionário.

Tabela 4 - Respostas do questionário.

\begin{tabular}{lcccccc}
\hline KPIs & $\boldsymbol{1}$ & $\mathbf{2}$ & $\mathbf{3}$ & $\mathbf{4}$ & $\sum$ & Importância \\
\hline KPI.1 & 0 & 0 & 5 & 16 & 21 & 0,9405 \\
KPI.2 & 0 & 0 & 9 & 12 & 21 & 0,8929 \\
KPI.3 & 0 & 0 & 11 & 10 & 21 & 0,8690 \\
KPI.4 & 0 & 0 & 10 & 11 & 21 & 0,8810 \\
KPI.5 & 0 & 0 & 7 & 14 & 21 & 0,9167 \\
KPI.6 & 0 & 3 & 12 & 6 & 21 & 0,7857 \\
KPI.7 & 0 & 2 & 12 & 7 & 21 & 0,8095 \\
KPI.8 & 0 & 0 & 13 & 8 & 21 & 0,8452 \\
KPI.9 & 0 & 0 & 14 & 7 & 21 & 0,8333 \\
KPI.10 & 0 & 0 & 4 & 17 & 21 & 0,9524 \\
KPI.11 & 0 & 0 & 13 & 8 & 21 & 0,8452 \\
KPI.12 & 0 & 0 & 10 & 11 & 21 & 0,8810 \\
KPI.13 & 0 & 0 & 12 & 9 & 21 & 0,8571 \\
KPI.14 & 0 & 1 & 8 & 12 & 21 & 0,8810 \\
KPI.15 & 0 & 0 & 9 & 12 & 21 & 0,8929 \\
KPI.16 & 0 & 0 & 2 & 19 & 21 & 0,9762 \\
\hline K 5 (1) & 0 & 019 & 21 \\
\hline
\end{tabular}

$1=$ sem importância; $2=$ pouco importante, $3=$ importante $; 4=$ muito importante

O cálculo da importância de cada KPI foi realizado mediante soma das multiplicações do número de respondentes 


\section{REVISTA}

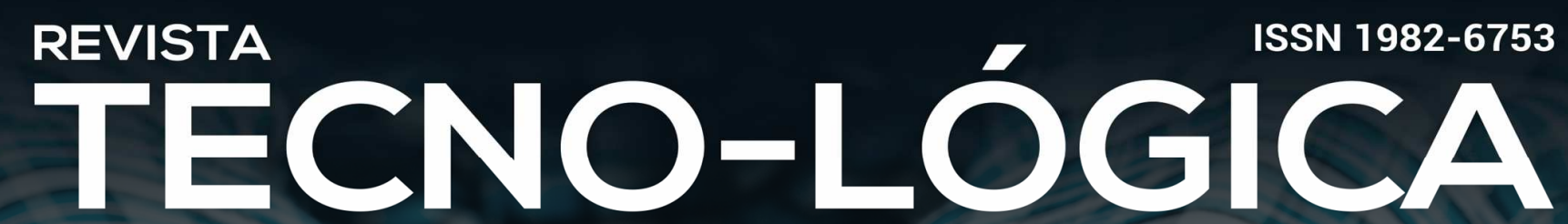

\section{Edição Especial com publicação de trabalhos selecionados do V SIGEPRO}

pelo peso em cada alternativa e dividido pelo número total de respondentes. Os KPI mais apontados como muito importante foram: custo do produto vendido (KPI 16), pontualidade (KPI 10) e qualidade do produto adquirido (KPI 1). Percebe-se que a economia está diretamente relacionada com a redução dos custos nas organizações, a pontualidade está alinhada com entregas dentro dos prazos e a qualidade é um fator intrínseco em qualquer instituição que visa lucro. Quanto aos indicadores que obtiveram os menores níveis de importância, encontram-se: flexibilidade de volume (KPI 6) e de entrega (KPI 7) e lead time (KPI 9). Nota-se que as empresas não realizam mudanças significativas em seus pedidos de compra, sendo que há pouca demanda de alterações em pedidos já realizados.

As respostas do questionário foram utilizadas no cálculo das taxas de substituição, que representam a importância das alternativas refletidas na árvore da gestão da cadeia de suprimentos. Com esse cálculo, foi possível identificar quais indicadores apresentados possuem maior/menor importância frente ao contexto em questão. Inicialmente, foi determinada a importância de cada KPI dentro de seu FCS. Na sequência, foram mensuradas as representatividades dos FCS e dos PVF. Por fim, definiu-se a importância dos PVF.

Utilizando o cálculo da importância de cada KPI, definiuse o valor da taxa de substituição desde os KPI, evoluindo para os FCS, PVF e concluindo com o cálculo da taxa global de substituição. Para o cálculo da taxa de substituição dos KPI (TSkpi), foi efetuada a multiplicação entre as médias e o valor percentual do KPI (KPI / FCS). A Equação 1 representa esse cálculo [21].

$$
T S k p i=\bar{R} \times \frac{1}{n k p i / f c s}
$$

TECNO-LÓGICA, Santa Cruz do Sul, v. 24, n. nesp, p. 275-288, jul./dez. 2020
Transformando cada taxa de substituição em percentual de representatividade em relação ao seu FCS, identifica-se quanto cada KPI representa de seu FCS. Na sequência, o cálculo da taxa de substituição dos FCS é realizado através da multiplicação do percentual que cada um representa pelo somatório das taxas de substituição dos KPI deste FCS. O cálculo da taxa de substituição do FCS foi efetuado conforme a Equação 2 [21].

$$
T S f c S=\sum_{i=1}^{n} \times T S k p i \times \frac{1}{(n k p i \text { do } p v f) \times(p v f / n k p i \text { do } f c s)}
$$

Da mesma maneira que foram calculados os percentuais dos KPI, foram calculados os percentuais dos FCS em relação aos PVF. Por fim, o último nível do cálculo visa encontrar as taxas de substituição dos PVF, que segue a lógica dos cálculos feitos anteriormente, onde a multiplicação do percentual que cada PVF representa é multiplicada pela soma dos valores encontrados na taxa de substituição dos FCS, conforme a Equação 3 [21].

$$
T S p v f=\sum_{i=1}^{n} \times T S f C S \times\left[\frac{1}{(\text { nkpi total }) \times(\text { mkpi do } p v f)}\right] \times 100
$$

Desse modo, todos os valores calculados podem ser observados na Tabela 5.

A matéria publicada nesse periódico é licenciada sob forma de uma Licença Creative Commons - Atribuição 4.0 Internacional http://creativecommons.org/licenses/by/4.0/ 


\section{REVISTA}

\section{Edição Especial com publicação de trabalhos selecionados do V SIGEPRO}

Tabela 5 - Taxa de substituição geral.

\begin{tabular}{|c|c|c|c|c|c|c|c|c|c|}
\hline$P V$ & & $R 1$ & $F C S$ & $R 2$ & KPI & $R 3$ & TSkpi & $T S f c s$ & $T S p v f$ \\
\hline \multirow{2}{*}{ PVF.1 } & \multirow{2}{*}{0,13} & 0,50 & FCS.1 & 1,00 & KPI.1 & 0,9405 & $\begin{array}{l}0,9405 \\
(100 \%)\end{array}$ & $\begin{array}{c}0,470 \\
(51,30 \%)\end{array}$ & \multirow{2}{*}{$\begin{array}{c}0,1146 \\
(13,06 \%)\end{array}$} \\
\hline & & 0,50 & FCS.2 & 1,00 & KPI.2 & 0,8929 & $\begin{array}{l}0,8929 \\
(100 \%)\end{array}$ & $\begin{array}{c}0,446 \\
(48,70 \%)\end{array}$ & \\
\hline \multirow{5}{*}{ PVF.2 } & \multirow{5}{*}{0,31} & 0,20 & FCS. 3 & 1,00 & KPI.3 & 0,8690 & $\begin{array}{l}0,8690 \\
(100 \%)\end{array}$ & $\begin{array}{c}0,174 \\
(20,39 \%)\end{array}$ & \multirow{5}{*}{$\begin{array}{c}0,2664 \\
(30,37 \%)\end{array}$} \\
\hline & & 0,20 & FCS.4 & 1,00 & KPI.4 & 0,8810 & $\begin{array}{l}0,8810 \\
(100 \%)\end{array}$ & $\begin{array}{c}0,176 \\
(20,67 \%)\end{array}$ & \\
\hline & & 0,20 & FCS.5 & 1,00 & KPI.5 & 0,9167 & $\begin{array}{l}0,9167 \\
(100 \%)\end{array}$ & $\begin{array}{c}0,183 \\
(21,51 \%)\end{array}$ & \\
\hline & & \multirow{2}{*}{0,40} & \multirow{2}{*}{ FCS.6 } & 0,50 & KPI.6 & 0,7857 & \multirow{2}{*}{$\begin{array}{c}(49,25 \%) \\
0,4048 \\
(50,75 \%)\end{array}$} & \multirow{2}{*}{$\begin{array}{c}0,319 \\
(37,43 \%)\end{array}$} & \\
\hline & & & & 0,50 & KPI.7 & 0,8095 & & & \\
\hline \multirow{3}{*}{ PVF.3 } & \multirow{3}{*}{0,19} & 0,33 & FCS.7 & 1,00 & KPI.8 & 0,8452 & $\begin{array}{l}0,8452 \\
(100 \%)\end{array}$ & $\begin{array}{c}0,282 \\
(32,13 \%)\end{array}$ & \multirow{3}{*}{$\begin{array}{c}0,1644 \\
(18,74 \%)\end{array}$} \\
\hline & & 0,33 & FCS. 8 & 1,00 & KPI.9 & 0,8333 & $\begin{array}{l}0,8333 \\
(100 \%)\end{array}$ & $\begin{array}{c}0,278 \\
(31,67 \%)\end{array}$ & \\
\hline & & 0,33 & FCS.9 & 1,00 & KPI.10 & 0,9524 & $\begin{array}{l}0,9524 \\
(100 \%)\end{array}$ & $\begin{array}{c}0,317 \\
(36,20 \%)\end{array}$ & \\
\hline \multirow{3}{*}{ PVF.4 } & \multirow{3}{*}{0,19} & 0,33 & FCS. 10 & 1,00 & KPI.11 & 0,8452 & $\begin{array}{l}0,8452 \\
(100 \%)\end{array}$ & $\begin{array}{c}0,282 \\
(32,72 \%)\end{array}$ & \multirow{3}{*}{$\begin{array}{c}0,1615 \\
(18,41 \%)\end{array}$} \\
\hline & & \multirow{2}{*}{0,67} & \multirow{2}{*}{ FCS.11 } & 0,50 & KPI.12 & 0,8810 & $\begin{array}{c}0,4405 \\
(50,68 \%)\end{array}$ & \multirow{2}{*}{$\begin{array}{c}0,579 \\
(67,28 \%)\end{array}$} & \\
\hline & & & & 0,50 & KPI.13 & 0,8571 & $\begin{array}{c}0,4286 \\
(49,32 \%)\end{array}$ & & \\
\hline \multirow{3}{*}{ PVF.5 } & \multirow{3}{*}{0,19} & \multirow{3}{*}{1,00} & \multirow{3}{*}{ FCS.12 } & 0,33 & KPI.14 & 0,8810 & $\begin{array}{c}0,2907 \\
(32,03 \%)\end{array}$ & \multirow{3}{*}{$\begin{array}{c}0,908 \\
(100 \%)\end{array}$} & \multirow{3}{*}{$\begin{array}{c}0,1702 \\
(19,41 \%)\end{array}$} \\
\hline & & & & 0,33 & KPI.15 & 0,8929 & $\begin{array}{c}0,2946 \\
(32,47 \%)\end{array}$ & & \\
\hline & & & & 0,33 & KPI.16 & 0,9762 & $\begin{array}{c}0,3221 \\
(35,50 \%)\end{array}$ & & \\
\hline
\end{tabular}

$R 1=F C S / P V F ; R 2=K P I / F C S ; R 3=$ KPI médio.

A partir dos dados de todas as taxas de substituição foi possível definir o ranking dos KPI, FCS e PVF em relação à sua taxa de substituição, gerando assim uma comparação baseada nos cálculos efetuados. Esses dados podem ser vistos nas Tabelas 6, 7 e 8 .
Tabela 6 - Ranking dos KPI

\begin{tabular}{llc}
\hline KPI & Descrição do KPI & TSkpi \\
\hline KPI.10 & OTD (On-time Delivery, entrega dentro do prazo estipulado) & 0,9524 \\
KPI.1 & Qualidade de produto adquirido & 0,9405 \\
KPI.5 & Saving (valor economizado na compra) & 0,9167 \\
KPI.2 & Defeito por unidade & 0,8929 \\
KPI.4 & Prazo médio de pagamento & 0,8810 \\
KPI.3 & Evolução de preço & 0,8690 \\
KPI.8 & Ponto de pedido & 0,8452 \\
KPI.11 & Devoluções & 0,8452 \\
KPI.9 & Lead time (tempo de entrega) & 0,8333 \\
KPI.12 & Pedido perfeito & 0,4405 \\
KPI.13 & OTIF (On-Time In Full, entrega atendendo todos os quesitos do pedido) & 0,4286 \\
KPI.7 & Flexibilidade de entrega & 0,4048 \\
KPI.6 & Flexibilidade de volume & 0,3929 \\
KPI.16 & Custo do produto vendido & 0,3221 \\
KPI.15 & Custo de manutenção do estoque & 0,2946 \\
KPI.14 & Custo de processamento dos pedidos & 0,2907 \\
\hline & &
\end{tabular}

Comparando com os dados de importância (Tabela 4), obteve-se um resultado similar. Os KPI 10 (pontualidade), 1 (qualidade) e 5 (saving) mantiveram-se entre os mais importantes, porém, o de número 16 não se encontra mais no topo. Isso se deve ao cálculo da taxa de substituição; o PVF 5 e o FCS 12 têm mais 2 indicadores em conjunto, além do custo do produto vendido. Fazendo, assim, com que as importâncias antes dadas para esses outros dois indicadores contribuam para que o KPI 16 tenha uma taxa de substituição inferior. 


\section{REVISTA}

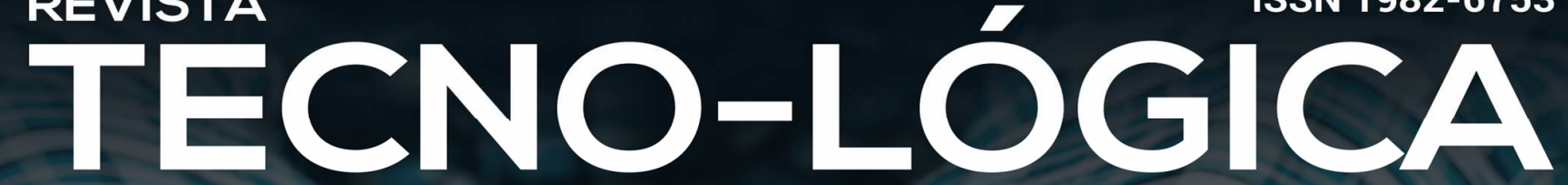

\section{Edição Especial com publicação de trabalhos selecionados do V SIGEPRO}

Tabela 7 - Ranking dos FCS

\begin{tabular}{llc}
\hline FCS & Descrição do $\boldsymbol{F C S}$ & TSf $\boldsymbol{c}$ \\
\hline FCS.12 & Custos Operacionais & 0,908 \\
FCS.11 & Acuracidade do atendimento do pedido & 0,579 \\
FCS.1 & Cumprimento das especificações técnicas de qualidade & 0,470 \\
FCS.2 & Menor número de defeitos & 0,446 \\
FCS.6 & Possibilidade de alteração do pedido de compra & 0,319 \\
FCS.9 & Pontualidade & 0,317 \\
FCS.7 & Reabastecimento do estoque & 0,282 \\
FCS.10 & Entrega sem avaria & 0,282 \\
FCS.8 & Tempo entre a solicitação de compra e o recebimento & 0,278 \\
FCS.5 & Valor economizado na compra & 0,183 \\
FCS.4 & Prazo de pagamento & 0,176 \\
FCS.3 & Sazonalidade de preço & 0,174 \\
\hline
\end{tabular}

No ranking dos FCS, Tabela 7, observou-se que os fatores mais importantes na visão dos gestores de compras são: custos operacionais, acuracidade do atendimento do pedido, cumprimento das especificações de qualidade e menor número de defeitos. Fica claro que as áreas de qualidade e custos vêm à tona com maior importância nesses fatores. Já outros, como sazonalidade de preço, prazo de pagamento e valor economizado na compra, tiveram taxas de substituição menores que os demais. Destaca-se que esses últimos três pertencem ao mesmo PVF, a flexibilidade.

Tabela 8 - Ranking dos PVF.

\begin{tabular}{lll}
\hline PVF & Descrição do PVF & TSpvf \\
\hline PVF.2 & Flexibilidade & 0,2664 \\
PVF.5 & Custos & 0,1702 \\
PVF.3 & Rapidez & 0,1644 \\
PVF.4 & Confiabilidade & 0,1615 \\
PVF.1 & Qualidade & 0,1146 \\
\hline
\end{tabular}

Por fim, obteve-se a classificação quanto aos PVF. Como pode ser observado no Tabela 8 , flexibilidade teve uma taxa de substituição superior quando comparada aos demais PVF - custos, rapidez, confiabilidade e qualidade.

\subsection{Cálculo da taxa global}

Após efetuados os cálculos das médias, das taxas de substituições dos PVF, FCS e KPI obteve-se os dados necessários para revelar as taxas de substituição global. Calculou-se a taxa global individual de cada KPI (Equação 4), que representa a multiplicação entre a taxa de substituição do PVF, FCS e KPI em questão. Em seguida, foram somadas todas as taxas globais individuais dos KPI e feito um ranking baseado na representatividade de cada KPI sobre a composição da modelagem como um todo [13].

$$
W K P I_{n}=P V F_{n} \times F C S_{n} \times K P I_{n}
$$

Com os cálculos de cada taxa global individual dos KPI efetuados, foi feita a soma dos respectivos valores para os PVF, visando abranger sua representatividade global. Com as 5 taxas globais dos PVF definidos, foi efetuada a soma para definir a taxa global de importância dos indicadores utilizados na pesquisa, que resultou em 0,6773 - Tabela 9.

Tabela 9 - Taxas globais dos KPI e PVF.

\begin{tabular}{|c|c|c|c|c|c|c|c|}
\hline \multicolumn{2}{|c|}{$P V F$} & KPI & TSkpi & $T S f c s$ & $T S p v f$ & TGkpi & $T G p v f$ \\
\hline \multirow{2}{*}{ PVF.1 } & \multirow{2}{*}{0,13} & KPI.1 & 0,9405 & 0,470 & \multirow{2}{*}{0,1146} & 0,0507 & \multirow{2}{*}{0,0963} \\
\hline & & KPI.2 & 0,8929 & 0,446 & & 0,0457 & \\
\hline \multirow{5}{*}{ PVF. 2} & \multirow{5}{*}{0,31} & KPI.3 & 0,8690 & 0,174 & \multirow{5}{*}{0,2664} & 0,0402 & \multirow{5}{*}{0,1941} \\
\hline & & KPI.4 & 0,8810 & 0,176 & & 0,0413 & \\
\hline & & KPI.5 & 0,9167 & 0,183 & & 0,0448 & \\
\hline & & KPI.6 & 0,3929 & \multirow{2}{*}{0,319} & & 0,0334 & \\
\hline & & KPI.7 & 0,4048 & & & 0,0344 & \\
\hline \multirow{3}{*}{ PVF.3 } & \multirow{3}{*}{0,19} & KPI.8 & 0,8452 & 0,282 & \multirow{3}{*}{0,1644} & 0,0392 & \multirow{3}{*}{0,1269} \\
\hline & & KPI.9 & 0,8333 & 0,278 & & 0,0381 & \\
\hline & & KPI.10 & 0,9524 & 0,317 & & 0,0497 & \\
\hline \multirow{3}{*}{ PVF.4 } & \multirow{3}{*}{0,19} & KPI.11 & 0,8452 & 0,282 & \multirow{3}{*}{0,1615} & 0,0385 & \multirow{3}{*}{0,1197} \\
\hline & & KPI.12 & 0,4405 & \multirow{2}{*}{0,579} & & 0,0412 & \\
\hline & & KPI.13 & 0,4286 & & & 0,0401 & \\
\hline \multirow{4}{*}{ PVF.5 } & \multirow{3}{*}{0,19} & KPI.14 & 0,2907 & \multirow{3}{*}{0,908} & \multirow{3}{*}{0,1702} & 0,0449 & \multirow{3}{*}{0,1401} \\
\hline & & KPI.15 & 0,2946 & & & 0,0455 & \\
\hline & & KPI.16 & 0,3221 & & & 0,0497 & \\
\hline & & & & & & 0,6773 & 0,6773 \\
\hline
\end{tabular}

A matéria publicada nesse periódico é licenciada sob forma de uma Licença Creative Commons - Atribuição 4.0 Internacional http://creativecommons.org/licenses/by/4.0/ 


\section{REVISTA}

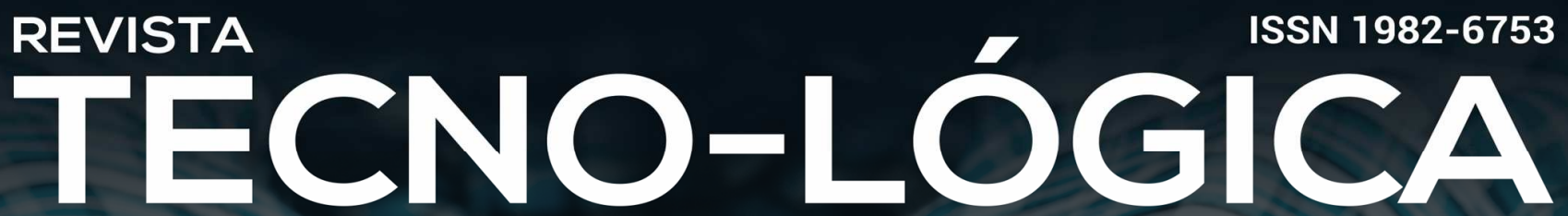

\section{Edição Especial com publicação de trabalhos selecionados do V SIGEPRO}

Analisando de forma individual cada KPI e considerando as taxas globais de substituição encontradas, identificou-se sua representatividade de modo geral, verificando sua porcentagem em relação ao valor da taxa geral encontrada, que foi de 0,6773 . Dessa maneira, é possível verificar a importância de cada um dos indicadores, de acordo com o Tabela 10.

Tabela 10 - Ranking da taxa global individual dos KPI.

\begin{tabular}{llc}
\hline KPI & Descrição do KPI & TGkpi \\
\hline KPI.16 & Custo do produto vendido & $6,89 \%$ \\
KPI.10 & OTD (On-Time Delivery) & $6,79 \%$ \\
KPI.1 & Qualidade de produto adquirido & $6,70 \%$ \\
KPI.5 & Saving & $6,53 \%$ \\
KPI.2 & Defeito por unidade & $6,36 \%$ \\
KPI.15 & Custos de manutenção do estoque & $6,30 \%$ \\
KPI.12 & Pedido perfeito & $6,28 \%$ \\
KPI.4 & Prazo médio de pagamento & $6,28 \%$ \\
KPI.14 & Custo de processamento dos pedidos & $6,22 \%$ \\
KPI.3 & Evolução de preço & $6,19 \%$ \\
KPI.13 & OTIF (On-Time in Full) & $6,11 \%$ \\
KPI.11 & Devoluções & $6,02 \%$ \\
KPI.8 & Ponto de pedido & $6,02 \%$ \\
KPI.9 & Lead time & $5,94 \%$ \\
KPI.7 & Flexibilidade de entrega & $5,77 \%$ \\
KPI.6 & Flexibilidade de volume & $5,60 \%$ \\
\hline
\end{tabular}

Na Tabela 10, observa-se a importância dada aos indicadores de custo do produto vendido $(6,89 \%$ da taxa global geral), OTD (6,79\%), qualidade do produto (6,53\%) e saving $(6,36 \%)$. Portanto, é possível constatar que, para um gestor de compras, fatores como custo, pontualidade e qualidade são mais representativos que os demais, pois impactam significativamente nos resultados da empresa. Na parte inferior da classificação, pode-se observar os mesmos indicadores de flexibilidade, que de acordo com os gestores, não possuem tanta importância quanto os demais. Os resultados obtidos nessa análise percentual final coincidem com as informações contidas na Tabela 4, que destacava as respostas das empresas multiplicadas pelas escalas adotadas no questionário, confirmando a veracidade dos cálculos efetuados.

Observou-se que alguns fatores possuem maior destaque quando se trata de indicadores de suprimentos como, por exemplo, qualidade, pontualidade e custos, sendo esses os escolhidos como muito importantes pelos gestores. Já os fatores que lidavam com flexibilidade foram julgados menos importantes. Dessa forma, foi possível determinar o ranking dos KPI desenvolvido a partir dos resultados do questionário e, após, pelas taxas de substituição e global.

Outra constatação, a partir dos resultados, foi a dificuldade em determinar quais indicadores são os mais apropriados para o controle de processos. Por consequência, prejudicando a seleção de qual método de pesquisa utilizar para a obtenção destes KPI. Com a definição dos KPI, os FCS foram determinados de acordo com o seu agrupamento, identificando os PVF, completando o fluxo da modelagem de indicadores. Esses são os pilares para a estruturação da árvore de decisões, que foi utilizada para o desenvolvimento da modelagem do sistema de avaliação de desempenho da cadeia de suprimentos. Ademais, constatou-se que a definição dos principais KPI teve resultados similares tanto diretamente na aplicação da survey quanto após o tratamento dos dados através das taxas de substituição e global, reforçando os resultados obtidos dos respondentes.

Por fim, a partir dos rankings estabelecidos, foram identificados os indicadores de maior importância, sendo eles: custo do produto vendido, OTD e qualidade do produto adquirido. Tratando-se dos FCS, foram definidos como mais importantes os custos operacionais, a acuracidade do atendimento do pedido e o cumprimento das especificações técnicas de qualidade. Quanto aos 


\section{REVISTA}

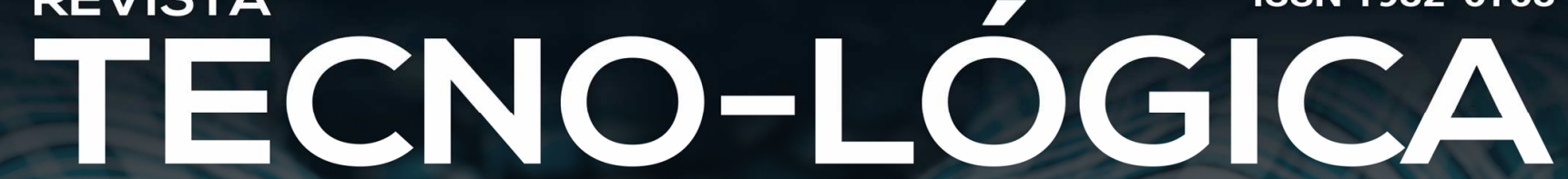

Edição Especial com publicação de trabalhos selecionados do V SIGEPRO

PVF, as melhores colocações foram qualidade, flexibilidade e custos.

\section{Considerações finais}

Com base nas colocações apresentadas a partir da modelagem construída, foi possível identificar quais indicadores têm maior impacto no setor de suprimentos, segundo os gestores de suprimentos das empresas contatadas. No entanto, se a empresa não possuir os dados necessários, a implantação desses indicadores fica prejudicada. Inclusive, fica a critério da empresa a escolha de quais indicadores deseja implantar em seu sistema.

Com a taxa global de substituição $(0,6773)$ e as taxas de substituição individuais de cada KPI definidas, foi possível observar quais são os indicadores que mais impactam no valor global. Dessa maneira, é possível trabalhá-los para a obtenção de um desempenho melhor no setor de compras da empresa. Ficou claro, também, que o indicador de flexibilidade do PVF teve maior importância, segundo dados obtidos do questionário.

Diante da taxa de respondentes da população consultada, 21 para 46, nota-se dificuldades de obtenção das respostas das empresas. Neste sentido, espera-se que pesquisas futuras possam abranger um número maior de empresas, suportando os resultados obtidos por esta pesquisa e tornando-a ainda mais assertiva. Além disso, este estudo pode ser adaptado para os setores de suprimentos de outros segmentos de produção.

Como contribuição desta pesquisa, pode-se ressaltar o delineamento proposto de indicadores-chave de desempenho, a partir da revisão da literatura e com o apoio dos gestores de cadeias de suprimentos. A utilização de um mapeamento embasado cientificamente pode produzir resultados mais assertivos no monitoramento desse setor, não apenas o de embalagens flexíveis, mas também em outros segmentos. Ademais, esta pesquisa pode ser replicada, ou até mesmo seguida como base na proposição de um sistema de avaliação de desempenho para outros segmentos da indústria. Com os indicadores bem definidos, uma empresa pode produzir vantagens competitivas frente a seus concorrentes, fator extremamente relevante em um cenário de mercado acirrado.

\section{Agradecimentos}

Os autores agradecem a Coordenação de Aperfeiçoamento de Pessoal de Nível Superior (CAPES) pelo suporte a esta pesquisa - Processos 88887.342073/2019-00 (Goecks, L. S.) e 88887.342081/2019-00 (Gomes, I. B.).

\section{SUPPLY CHAIN SECTOR INDICATORS: AN EXPLORATORY STUDY IN FLEXIBLE PACKAGING MANUFACTURING INDUSTRIES}

ABSTRACT: Several companies find it difficult to maximize the potential of their supply chain as they fail to develop an effective evaluation method. Given this problem and the necessity to effectively measure the supply chain, this study aims at identifying the performance indicators. The study was structured from a bibliographic review and a questionnaire to identify the main indicators of the supply chain, which was sent to flexible packaging companies in the state of Rio Grande do Sul. Based on the results and calculations of the Key Performance Indicators (KPI), Critical Success Factors (CSF) and Fundamental Viewpoints (FV), rankings were defined according to the questionnaire. For KPIs, the most important indicators were: cost 


\section{REVISTA}

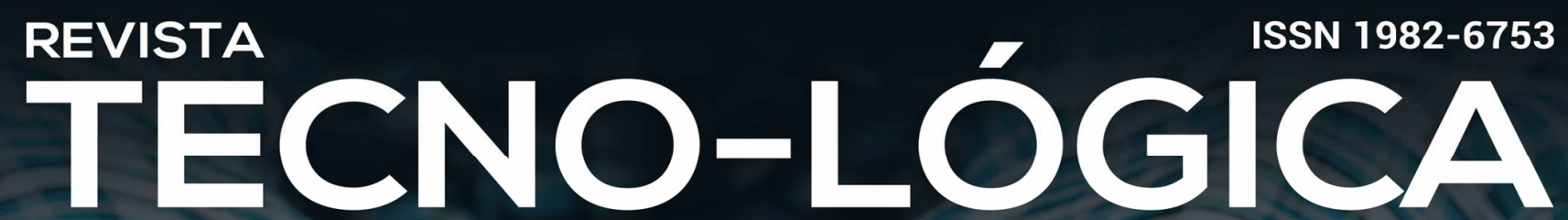

\section{Edição Especial com publicação de trabalhos selecionados do V SIGEPRO}

of the product sold, on-time delivery (OTD) and quality of the purchased product. In relation to the CSF, operational costs, purchase order accuracy and compliance with technical specifications of quality were defined as the most important. Regarding to the FV, the best placements were quality, flexibility and costs. Finally, this study contributes to the proposition of key performance indicators, based on the literature and supported by supply chain managers.

Keywords: Supplies. Indicators. Flexible Packaging. Survey.

\section{Referências}

[1] ZHANG, H.; OKOROAFO, S. C. Third-party logistics (3PL) and supply chain performance in the Chinese market: a conceptual framework. Engineering Management Research, Vol. 4, n. 1, p. 38-48, 2015.

[2] SHAW, S.; GRANT, D. B.; MANGAN, J. Developing environmental supply chain performance measures. Benchmarking: An International Journal, Vol. 17, n. 3, p. 320-339, 2010.

[3] MUYSINALIYEV, A.; AKTAMOV, S. Supply chain management concepts: literature review. IOSR Journal of Business and Management, Vol. 15, n. 6, p. 60$66,2014$.

[4] GUNASEKARAN, A.; KOBU, B. Performance measures and metrics in logistics and supply chain management: a review of recent literature (1995-2004) for research and applications. International Journal of Production Research, Vol. 45, n. 12, p. 2819-2840, 2007.

[5] MENTZER, J. T.; DEWITT, W.; KEEBLER, J. S.; MIN, S.; NIX, N. W.; SMITH, C. D.; ZACHARIA, Z.G. Defining supply chain management. Journal of Business Logistics, Vol. 22, n. 2, p. 1-25, 2001.

[6] CHRISTOPHER, M. Logistics and supply chain management. 5. ed. Pearson: United Kingdom, 2016, 328p.

[7] CHORFI, Z.; BERRADO, A.; BENABBOU, L. Selection of key performance indicators for supply chain monitoring using MCDA. In: 10th International Conference on Intelligent Systems: Theories and Applications, 2015, Rabat. Anais... SITA - IEEE, 2015, p. 1-6.
[8] AKYUZ, G. A.; ERKAN, T. E. Supply chain performance measurement: a literature review. International Journal of Production Research, Vol. 48, n. 17, p. 5137-5155, 2010.

[9] CAI, J. LIU, X.; XIAO, Z.; LIU, J. Improving supply chain performance management: A systematic approach to analyzing iterative KPI accomplishment. Decision Support Systems, Vol. 46, n. 2, p. 512-521, 2009.

[10] STANDING, C; CRIPPS, H. Critical success factors in the implementation of electronic health records: A two-case comparison. Systems Research and Behavioral Science, Vol. 32, n. 1, p. 75-85, 2015.

[11] ESTEVES, J.; PASTOR-COLLADO, J. Analysis of critical success factors relevance along SAP implementation phases. In: Americas Conference on Information Systems - AMCIS 2001 Proceedings, p. 197, 2001.

[12] GIFFHORN, E. Construção de modelo de avaliação de desempenho de empresas terceirizadas com a utilização da metodologia MCDA-C. 2007, 258 f. Dissertação (Programa de Pós-Graduação em Engenharia de Produção - Mestrado) - Universidade Federal de Santa Catarina, Florianópolis, 2007.

[13] DICKEL, D. G. Modelagem e mensuração da inovação no setor de construção naval e offshore Brasileiro. 2015, 127 f. Dissertação (Programa de Pós-Graduação em Engenharia de Produção - Mestrado) - Universidade Federal de Santa Maria, Santa Maria. 2015.

[14] ALMEIDA, A. T. Processo de decisão nas organizações. Atlas: São Paulo, 2013, 231p.

[15] GOMES, C.F.; GOMES, L.F.A.M. Tomada de decisão gerencial: Enfoque Multicritério. 4. ed. Atlas: São Paulo, 2012.

[16] GIL, A. C. Como elaborar projetos de pesquisa. 6. ed. Atlas: São Paulo, 2017.

[17] CARNEVALLI, J. A.; MIGUEL, P. A. C. Desenvolvimento da pesquisa de campo, amostra e questionário para realização de um estudo tipo survey sobre a aplicação do QFD no Brasil. In: Encontro Nacional de Engenharia de Produção, 2001, Salvador. Anais... ENEGEP, 2001.

[18] THAYER-HART, N.; DYKEMA, J.; ELVER, K.; SCHAEFFER, N. C.; STEVENSON, J. Survey fundamentals: A guide to designing and implementing surveys. University of Wisconsin, 2010.

[19] SCC, Supply Chain Council. Supply-chain operations reference - model. versão 6.0. SCC: Pittsburgh, 2003. 


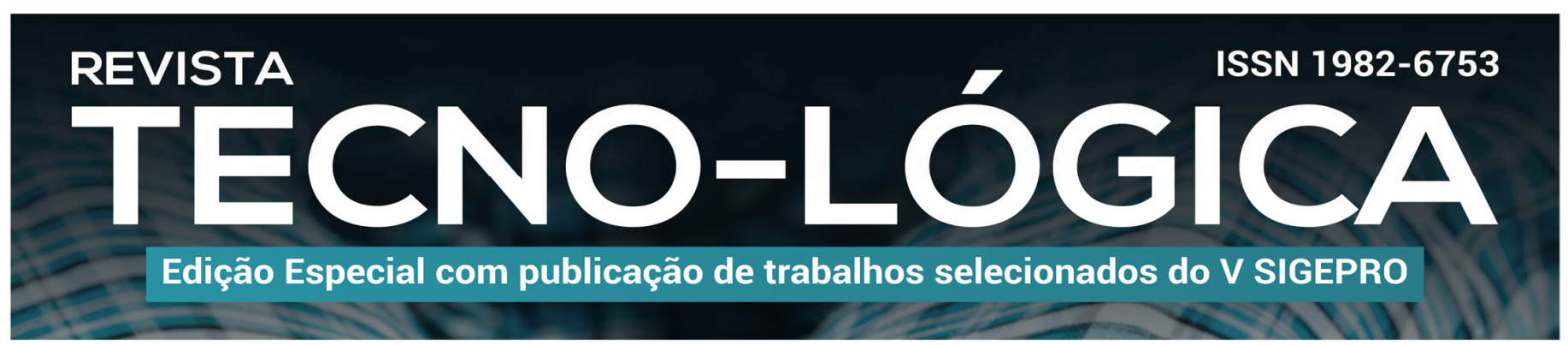

[20] AMBE, I. M. Key indicators for optimising supply chain performance: the case of light vehicle manufacturers in South Africa. Journal of Applied Business Research, Vol. 30, n. 1, p. 277-290, 2014.

[21] WATTE, E. F. Taxa global de competitividade para hospitais filantrópicos do Rio Grande do Sul. 2017. Dissertação (Programa de Pós-Graduação em Sistemas e Processos Industriais - Mestrado) - Universidade de Santa Cruz do Sul, Santa Cruz do Sul, 2017. 